Lep. Rev. (1966), 37, I, 7/15

\title{
Classification of Leprosy
}

\author{
D. L. LEIKER \\ Dermatologist, Institute of Tropical Hygiëne, Amsterdam, \\ formerly specialist leprologist, Ministry of Health, $\mathcal{N}$. . Vigeria
}

The three latest International Congresses of leprologists (Madrid r953, Tokio r958, Rio I 963 ) have recognised two polar types of leprosy, tuberculoid and lepromatous and one intermediate group, borderline.

These three categories faintly reflect the concept of a spectrum of clinical, immunological, bacteriological and histological signs.

Apart from these categories a group of cases with incharacteristic macular lesions, not fitting into the spectrum, called indeterminate, is recognised.

Although the basic concept of this classification is now almost generally accepted, few workers are really satisfied with its application in practice. Not a few workers have difficulties in fitting certain types of patients into this classification. Often there is agreement about the right place of patients in the spectrum, but disagreement about the designation.

The result is that considerable differences in type distribution are reported from the same area by different workers. Reports are difficult to compare unless one is familiar with the views of the investigator about classification.

The greatest differences are found in the proportions of indeterminate and 'intermediate' patients.

The less experienced worker is confused by designations such as reactional tuberculoid and tuberculoid in reaction, indeterminate and intermediate, dimorphous and borderline, which cover part of the same field but are not interchangeable. It can hardly be denied that the situation in the field of classification is still highly confusing for the average fieldworker in leprosy and rather indigestible for the general practioner who has to deal with leprosy only incidentally and who has no time nor need for a special study of the subject.

Much can be said for a simple classification for the lay-worker and a more detailed classication for the scientific worker. It is however doubtful that a simple classification can be designed without loss of significance. Classification should give information about important items such as infectiousness, complications to be expected, duration of treatment needed, ultimate prognosis, etc.

A division between open and closed cases has only momentary value. The open case of today may be a closed case tomorrow, and the reverse.

A division between benign and malign is an unsatisfactory substitute for cases on the tuberculoid side and on the lepromatous side. Many tuberculoid patients truly are benign, but many of the most severely crippled patients also belong to the tuberculoid category. Lepromatous leprosy, called malign, may be present for a decade or more, without serious complications and without producing deformity.

The fact that leprosy patients present an uninterrupted scale of all degrees of tissue resistance to Myco leprae means that each grouping is arbitrary and a compromise. One should not expect sharply defined groups.

It is not logical to distinguish between tuberculoid and lepromatous types and borderline and indeterminate groups. In fact all categories are groups. At most one could speak of patients as tuberculoid and lepromatous polar types, e.g. the small, single, rapidly selfhealing typical tuberculoid lesion and the pure, primary, diffuse lepromatous case respectively.

It is also not logical to divide the scale into a very large tuberculoid group, a very small borderline group and a large lepromatous group. The large groups thus become very heterogenous, whereas the borderline group is restricted to a small, rather well defined section of a large intermediate (dimorphous) group.

Nor is it logical to classify together a large number of macular lesions with an entirely different evolution, but which cannot be fitted into the scale at first glance, into an indeterminate 'dustbin'. 


\begin{tabular}{|c|c|c|c|c|c|c|c|}
\hline \multicolumn{8}{|c|}{ Onset } \\
\hline \multicolumn{8}{|c|}{ Indeterminate group } \\
\hline $\begin{array}{l}\text { tuberculoid } \\
\text { inoculation } \\
\text { lesions }\end{array}$ & $\begin{array}{l}\text { High } \\
\text { resistant } \\
\text { tuberculoid }\end{array}$ & $\begin{array}{l}\text { Low } \\
\text { resistant } \\
\text { tuberculoid }\end{array}$ & $\begin{array}{l}\text { Borderline } \\
\text { tuberculoid }\end{array}$ & $\begin{array}{l}\text { Borderline } \\
\text { lepromatous }\end{array}$ & $\begin{array}{l}\text { Non- } \\
\text { diffuse } \\
\text { lepromatous }\end{array}$ & $\begin{array}{l}\text { Diffuse } \\
\text { lepromatous }\end{array}$ & $\begin{array}{l}\text { Pure, } \\
\text { primary } \\
\text { diffuse } \\
\text { lepromatus }\end{array}$ \\
\hline 'ТТТ & $\mathrm{TT}$ & $\mathrm{T}$ & $\mathrm{TB}$ & $\mathrm{BL}$ & $\mathrm{L}$ & LL & LLL \\
\hline varieties & $\begin{array}{l}\text { macular } \\
\text { minor } \\
\text { major }\end{array}$ & $\begin{array}{l}\text { macular } \\
\text { minor } \\
\text { major }\end{array}$ & $\begin{array}{l}\text { maculoid } \\
\text { minor } \\
\text { major }\end{array}$ & $\begin{array}{l}\text { maculoid } \\
\text { infiltrated } \\
\text { nodular }\end{array}$ & $\begin{array}{l}\text { macular } \\
\text { infiltrated } \\
\text { nodular }\end{array}$ & $\begin{array}{l}\text { macular } \\
\text { infiltrated } \\
\text { nodular }\end{array}$ & \\
\hline natural course & & selfh & aling $\leftarrow$ & $\rightarrow$ progr & ssive & & \\
\hline $\begin{array}{l}\text { Mitsuda } \\
\text { relapse } \\
\text { nerve } \\
\text { destruction }\end{array}$ & $\begin{array}{l}>8 \mathrm{~mm} \\
\text { seldom, } \\
\text { always early }\end{array}$ & $\begin{array}{l}5-8 \mathrm{~mm} \\
\pm \\
\text { often, } \\
\text { early }\end{array}$ & $\begin{array}{l}3-5 \mathrm{~mm} \\
+ \\
\text { often, } \\
\text { early }\end{array}$ & $\begin{array}{l}2-4 \mathrm{~mm} \\
++ \\
\text { often, } \\
\text { later }\end{array}$ & $\begin{array}{l}\mathrm{i}-3 \mathrm{~mm} \\
+++ \\
\text { less often, } \\
\text { late }\end{array}$ & $\begin{array}{l}0 .-2 \mathrm{~mm} \\
++++ \\
\text { not often, } \\
\text { very late }\end{array}$ & \\
\hline
\end{tabular}

In the author's opinion there are advantages in dividing thescale into more than threecategories. This does not necessarily make classification more difficult, but probably even more easy. A six group classification which is based upon the degree of the tuberculoid and lepromatous element does not require more insight in these elements than a three group classification does. The advantage is that if an error in classification is made in the three groups classification the consequences are serious but an error of a shift of one group to the tuberculoid or to the lepromatous side is less serious in a six group classification.

In this article no fundamental changes in the present classification are suggested. A solution is sought in a further subdivision of the scale.

For the time being, the present terminology, although not always satisfactory, is preferred to a change. The introduction of new designations may lead to further confusion and to sterile discussions. The principle behind the designation is of far greater importance than the name.

\section{Indeterminate leprosy}

Per de

stable, seldom bacteriologically positive, presenting flat skin lesions. The reaction to lepromin is negative or positive. Neuritic complications may develop. These patients evolve to the lepromatous type or to the tuberculoid type or may remain unchanged indefinitely (Technical Resolutions Madrid Congress r 953).
Not a few workers classify as indeterminate a large proportion of all cases which at the moment of examination present pale, flat macules only. Other workers classify only a few patients as indeterminate.

The author belongs to the latter.

Typical tuberculoid patients seldom if ever start as indeterminate. Macular tuberculoid does not need to be classified as indeterminate if due attention is being paid to definition, degree of hypopigmentation, hypoaesthesia, etc.

Diffuse lepromatous leprosy also does start as indeterminate leprosy. The individual macules may resemble indeterminate lesions, but number and distribution of the macules and the presence of positive smears of both ears are sufficiently characteristic for a lepromatous classification.

A large proportion of the 'low resistant tuberculoid' patients however start with indeterminate lesions. This is also true for borderline and for non-diffuse lepromatous leprosy. However the true nature of the condition soon becomes evident and soon the cases can be reclassified.

These three categories in which a temporary classification of indeterminate can not always be avoided from only a small proportion of the cases and they are not a sufficient explanation for the high percentages of indeterminate cases reported.

The percentage of indeterminate leprosy becomes high if quiescent and arrested patients are included. Typical tuberculoid lesions become flat, somewhat less well defined and less hypopig- 
mented. The same is true for low-resistant tuberculoid and borderline patients.

If however due attention is paid to history of the lesions definition of edges, degree of hypopigmentation, surface texture (atrophy), loss of hair, loss of perspiration, degree of hypoaesthesia, etc., it is possible to decide in a large proportion of the cases whether they are at the tuberculoid or at the lepromatous side. Lesions which have healed spontaneously but after one or more years still leave conspicuous residual hypopigmentation, atrophy and hypoaesthesia and are still clearly defined are not inderterminate but obviously at the tuberculoid side. Patients with considerable nerve damage and no signs of lepromatous or borderline lepromatous leprosy also are at the tuberculoid side because the nerve damage is a sign of tissue resistance.

In our opinion the classification of indeterminate should be restricted to patients with flat lesions, not numerous, ill defined, only slightly hypopigmented, without conspicuous atrophy, little loss of hair, perspiration or sensation, without serious damage to the larger nerves and without a history of elevation of the lesions.

The lepromin reaction is weak, not negative, but also not strongly positive. Bacilli are absent or scanty on routine examination.

Thus indeterminate leprosy becomes the first stage towards a large group of cases in between typical tuberculoid and diffuse lepromatous, with a serious prognosis if not treated or inadequately treated, because of the chances of relapse and deformities.

In many instances the classification will be a temporary one. In a smaller proportion of the patients, those who heal after treatment before dissemination of the bacilli and the appearance of more and more characteristic lesions, the classification of indeterminate is permanent.Even in these patients it is often possible to determine the place in the spectrum with the aid of the lepromin test.

The difficulty of classifying cases who have not been seen in the early or active stage is fully realised, but it is regarded as more advantageous to classify these patients with or without the aid of the lepromin test, as well as possible in the spectrum, rather than to include all such patients in an indeterminate hybrid group. The chance of essential errors is reduced by a six group classification.

\section{The tuberculoid group}

This group comprises cases which are truly benign and others which show a temporary tendency to progression and which have a much more serious prognosis because of the chance of deformities. It is possible to divide the group into a subgroup with very high resistance and a subgroup with lower resistance, high resistant or typical tuberculoid leprosy and low resistant tuberculoid leprosy.

High resistant tuberculoid leprosy presents the typical features of high resistance such as restriction of number and size of lesions, conspicuous asymmetrical distribution, relatively marked hypopigmentation, very sharp definition of the edges, papular surface or central healing with a narrow papular edge, relatively marked hypoaesthesia, loss of hair and loss of perspiration. In a minority of the cases, those who are rapidly self healing, the changes in the dermis are minimal and the papular structure is hardly visible. Such patients with one or a few small lesions can be classified as macular tuberculoid. In our experience most macular tuberculoid cases have shown changes in the surface texture and are secondary. The other patients may be divided according to the degree of infiltration and consequently to the degree of elevation into a minor and a major variety.

Typical tuberculoid leprosy is a localised process and most lesions probably indicate the site of inoculation. In a small number of the patients bacilli escape and some secondary skin lesions may be produced. Occasionally a few bacilli reach one or two of the larger nerves. Because of the high tissue resistance such a nerve may be early and seriously damaged, but the nerve involvement is localised (small section of the nerve only) and is as a rule asymmetrically distributed.

The lepromin reaction is strongly positive. Bacilli are not found on routine examination. Relapse seldom if ever occurs.

Low resistant tuberculoid leprosy presents predominating tuberculoid features but some of these signs are less marked and they may be even absent in part of the lesions.

The first lesions may be indeterminate or tuberculoid. In the latter case the lesion often is not typical tuberculoid. The whole lesion may be slightly raised, micropapular, only slightly hypopigmented, without much loss of hair, perspiration or sensation and part of the lesion may be

$$
\text { Classification of Leprosy }
$$


somewhat less well defined. Satellitc lesions are common. Some lesions show a weak tendency to central healing but the papular edge remains broad. Sometimes, but not always, a single lesion causes one to suspect low-resistant tuberculoid development, because of the broad edge and the presence of satellite lesions.

Healing of the lesions is slower than in typical tuberculoid patients. In untreated cases more lesions appear, sometimes only one crop, in other cases successive crops.

The number of lesions may be great, but the distribution on the trunk remains asymmetrical. The peripheral parts of the body are usually more effected than the trunk and the lesions show a strong tendency to symmetry. Very often the central part of the face is completely covered with a large lesion or with multiple, coalescing lesions. A butterfly shape on nose and cheeks is often seen. The ear regions are often affected. On the extremities lesions are very often found on both elbows and hands and both knees and feet.

In patients with numerous lesions the lesions on the trunk may also show a tendency to symmetry.

Frequently lesions are found at sites which usually are not affected in typical tuberculoid cases, e.g. scalp, ears, nose, chin, palms of hands, soles of feet and genitals.

The larger nerves frequently become involved. Both sides of the body are affected, and in each extremity several or all larger nerves are involved. The nerves are affected over a great length. Consequently the deformity is symmetrically distributed. Although the deformity often develops less acutely than in the typical tuberculoid patients the deformity is of ten very severe ('beggar-type').

The lepromin reaction is positive but not strongly so. Bacilli may be found in the active stages but are not numerous. They are often found in sections in small numbers in the nerve twigs. Spontaneous arrest is the rule but relapses are not uncommon. Deterioration towards the lepromatous side I have never observed.

Low resistant tuberculoid leprosy logically fits in between typical tuberculoid leprosy and borderline leprosy. The distinction has practical value because of the much more serious prognosis and the need for special precautions in the treatment.

Io Leprosy Review

\section{The borderline group}

If the criteria for borderline of the International Congresses are rigidly applied only a very low proportion of the patients can be classified as borderline. If however also patients with multiple macular, maculoid and slightly raised lesions which are not typically tuberculoid, nor typical lepromatous, are included, a logical place for these atypical patients is found. This seems to be permitted as the lepromin reaction in these cases is of the same order as in typical borderline cases and the course of the disease corresponds with the size of the lepromin reaction.

It is not ciear which mechanism is responsible for macular, minor, and major tuberculoid lesions. It probably is a factor localised in the skin or a presensitizing factor. It is certain that these are clinical varieties which do not fit into the spectrum of general resistance.

Similarly present classical borderline, with the infiltrated lesions corresponds with a major variety, but for the minor and macular or maculoid varieties no place is reserved in the classification. This has lead to the introduction of the controversal use of macular dimorphous, infiltrated dimorphous being similar to classical borderline. In the authors opinion this principle deserves preservation but a more neutral terminology may bridge the gap between difference of opinion.

Some patients, called borderline tuberculoid, in which the signs are more at the tuberculoid side, heal spontaneously, although slower than low resistant tuberculoid cases and often only after relapses. They do not become lepromatous. Bacteriology they are more often and longer positive than low resistant tuberculoid patients. Globi are not found. The lepromin reaction is positive but only weakly so, weaker than in low resistant tuberculoid cases. Bacilli often persist in biopsy sections in nerves when the skin lesions have become quiescent and the bacilli have disappeared from the infiltrations.

The nerve involvement is comparable with low resistant tuberculoid leprosy, but in cases without reactions the deformity develops slower and often is less severe. There is little difference in reactive patients.

Borderline tuberculoid leprosy in symptoms and course resembles low resistant tuberculoid leprosy, but evolution and regression are slower. The lesions become more numerous, particularly 
on the trunk, and the distribution of lesions is more symmetrical, without becoming completely symmetrical.

Hypopigmentation, loss of hair, of perspiration and of sensation are on the average less marked than in tuberculoid cases. The lesion appear to be somewhat less well defined. Such differences however are more difficult to assess for the less experienced worker than the absence of a papular surface structure and the absence of typical central healing.

The lesions are equally raised throughout the lesion or there may be a tendency to central regression, but complete central healing while the edge is still active is uncommon. In low-resistant tuberculoid leprosy the zone of greatest activity is at the edge, whereas in borderline tuberculoid leprosy the zone of greatest activity is between the centre and the edge. An active lesion may show some depression in the centre and the most infiltrated part slopes away to the edges. This part appears as a hypopigmented halo around the lesion. The slope however is rather steep and visible only after close inspection.

In some cases the first lesion or lesions become "immune areas"

Symptoms and course of borderline lepromatous leprosy more resemble lepromatous leprosy, but the lesions appear to be better defined, hypopigmentation is more marked and lasts longer than in lepromatous patients.

The lesions are often dome-shaped, without papular structure, without central healing. The hypopigmented halo is more plainly visible and broader. The slope away at the edges is less steep than in borderline tuberculoid patients. The lesions appear to be less well defined, but the skin in between lesions is normal.

In reactive patients the zone of greatest activity is the most infiltrated central part of the lesion. After reaction the centre may regress more rapidly than the periphery, suggesting central healing. The central healing however is not complete. Immune areas, also those surrounded by fresh infiltration during a phase of reactivity, are more often seen than in borderline tuberculoid patients, but they are present only in a minority of them.

In borderline tuberculoid leprosy it appears that the area is more immune than in borderline lepromatous cases. In the latter not seldom streaks of fresh infiltration or nodules are seen in the immune area.

The lesions are positive, often strongly so, but large globi are uncommon. The lepromin reaction is doubtful.

The nerves are affected as in borderline tuberculoid, but in patients without reactions the deformity develops slower.

Ups and downs are common, but ultimately the disease is progressive. Although cases may develop far to the lepromatous side, the borderline element can still be recognised in far advanced cases by the definition of the lesions, the presence of hypopigmentation, and the presence of areas of practically normal skin in between extensive infiltration.

\section{The lepromatous group}

Not all lepromatous patients completely lack resistance to Myco. leprae. The resistance is shown by a weak response to lepromin, never to a positive degree but still not entirely negligible. There are also clinical signs of resistance corresponding with the size of the lepromin reaction.

In non diffuse lepromatous leprosy the disease usually starts with clearly visible hypopigmented macules. They may even last until the disease becomes quiescent. The first macules are indeterminate, but soon the lepromatous classification can be based on the distribution of the macules and the positive smears from both ears. Gradually the macules become infiltrated but the lesions remain somewhat apparent until they coalesce. Nodules may appear in a relatively early stage.

In diffuse lepromatous patients the macules are hardly visible and when they are recognised they are already numerous and the distribution is typically lepromatous.

Residual hypopigmentation is not seen. The edges of the infiltration are too vague to be defined. The lepromin reaction is negligible.

\section{ISCUSSION}

At the uttermost tuberculoid end of the scale a group of 'inoculation lesions' may be recognised. Such lesions appear in individuals with a very high potential resistance to $M$. leprae, who have not yet developed their maximum of effective resistance at the time of infection. In this period they may present papular, papulo-nodules, or lichenoid, small macular lesions or small minor 
tuberculoid lesions. Such lesions most likely indicate the site of infection. They heal rapidly and spontaneously. Relapses are not seen, not even after intense and prolonged contact with open cases. The lepromin reaction rapidly becomes strongly positive.

Lara has studied these lesions in detail in young children. The author has found comparable lesions in many adults in New Guinea and Nigeria.

At the uttermost lepromatous end of the scale one could place the pure, primary diffuse lepromatous cases, well described from South America but not rare also in other countries. The author has however never seen the Lucio phenomenon in such patients outside South America.

Although nearly the whole skin is affected, there is no visible hypopigmentation nor infiltration. The first visible symptom is a somewhat congested face, more due to edema than to cellular infiltration, and also there may be some loss of eyebrows. Smears are already strongly positive in this stage. Lepromin reaction is virtually nil.

More common are the cases with very diffuse but better visible infiltration. Such cases are classified as diffuse lepromatous leprosy.

Reactional tuberculoid patients are not classified separately. This is a mixed group of patients, some of which are bacteriologically negative or scanty positive, who heal spontaneously after one or two crops of fresh lesions appear (and the lesions are clearly at the tuberculoid side). Such patients are now classified as minor or major low resistant tuberculoid, according to the degree of infiltration. Some patients are less clearly tuberculoid; they may be progressive, presenting several crops of lesions and they are more often positive, sometimes fairly strongly so. These patients are classified as minor or major borderline tuberculoid.

A few patients are progressive, strongly positive and deteriorate towards the lepromatous side. Such patients are included in the borderline lepromatous group.

It is questionable that evolution from major tuberculoid via reactional tuberculoid towards lepromatous really occurs. It seems more likely, that the first major lesion merely was the first stage of a low resistant tuberculoid or a borderline tuberculoid leprosy but that we have failed to recognise the relatively small differences which cannot always be seen in a single or a few lesions. Particularly in reactive lesions criteria such as degree of hypopigmentaion, definition of edges, presence of papules, etc., are difficult to assess.

This concept is supported by the finding that progression was never observed in patients with major tuberculoid like lesions with a strongly positive lepromin reaction but was seen in patients with lesions which looked like tuberculoid lesions but who responded weakly to lepromins. On closer inspection it was often found that the lesions were not as typically tuberculoid as the first impression suggested, but that signs such as satellite lesions, sloping away of edges, hypopigmented halo, surface texture, etc., indicated their true nature.

Maculo-anaesthetic leprosy may be divided into a large number of truly benign cases with single or few lesions and a smaller number of more progressive patients, often with serious nerve involvement.

It appears that most benign patients with single or few small lesions can be classified as macular tuberculoid. The fact that the epitheloid foci are minimal and often are found only in serial sections is not a strong argument against a tuberculoid classification. In patients with very few bacilli and a strong resistance to Myco. leprae one does not always expect the development of large tuberculoid foci. Also patients are often biopsied after the short stage of greatest activity.

In a minority of the maculo-anaesthetic patients the course of the disease closely resembles low resistant tuberculoid leprosy. The distribution of lesions is similar. The lepromin reaction is weakly positive. Deterioration towards lepromatous is not seen. Such patients are classified as macular low resistant tuberculoid.

Browne has mentioned macular patients which are temporarily strong positive, even with globi, and are neither tuberculoid nor lepromatous. I have seen only few cases which correspond with the description. No true globi, but large bunches of bacilli only were found. One wonders if such cases fit into a macular borderline tuberculoid group.

Dimorphous leprosy is a hybrid group between tuberculoid and lepromatous, not covered by the term borderline. Some cases are more at the tuberculoid end, others are more at the lepromatous end of the spectrum, some are self hea ting, 
others are progressive. The group comprises cases which are macular, maculoid, and more or less infiltrated. The principle of a larger intermediate group is endorsed. The dimorphous group is subdivided, using a less controversial terminology.

Most if not all dimorphous cases are preceded by an indeterminate stage.

Polyneuritic leprosy is not recognised as a separate group or as a subgroup.

It cannot be denied, that primary mononeuritic leprosy may exist, but on the other hand the possibility that the neuritis was preceded by an inoculation lesion, which has healed rapidly and has left a non specific scar only or no residual signs at all, is difficult to exclude.

By chance even a few cases with affection of more than one nerve may occur, but such cases should be rare.

More difficult to evaluate are reports about primary polyneuritic lesions followed by later appearance of skin lesions and deterioration. It is difficult to conceive how a blood or lymph dissemination of bacilli first produces multiple neuritic lesions without skin lesions and that skin lesions are produced after the second dissemination.

One wonders if there had not been vague, self healing skin lesions, not noticed by the patient or not connected with the disease which were inconspicuous or not visible any more at the time of examination. This concept is supported by the finding that in many (macular) low-resistant tuberculoid cases and even more in borderline tuberculoid cases the first skin lesions may disappear virtually completely. Often at most some dry patches or a minimal loss of sensation is found and sometimes not even that. The author has found in Nigeria, that in a rather high proportion of patients with polyneuritic lesions who first strongly deny previous skin lesions it is possible to detect traces of former lesions or a history of skin lesions can be obtained. In some cases the skin lesions were not noticed by the patient. It is likely that most polyneuritic cases are secondary polyneuritic.

It is often difficult to distinguish between borderline and low resistant tuberculoid in arrested patients with uncharacteristic residual skin lesions. This could be an argument for a special polyneutritic group or a subgroup of indeterminate leprosy. Here again however the danger of such a group becoming a 'waste paper basket' for all polyneuritic cases exists. The presence of serious deformity and the absence of visible or anamnestic signs of lepromatous leprosy, points to the tuberculoid side. It is proposed to classify these patients as low resistant tuberculoid, although some may be borderline tuberculoid. This error in classification is not regarded as serious. A more accurate classification is some times possible with the aid of the lepromin test.

One may ask whether a subdivision of borderline and of leproma tous leprosy has any practical significance. In the author's opinion the 'point of no return', regression of progression, is to be found between borderline tuberculoid and borderline lepromatous. It is possible that fluctuations in resistance occur during life, but there is little evidence that such fluctuations are great. Changes, if they occur, are probably limited to the order of one subgroup and only exceptionally go beyond a subgroup.

Secondly, in the borderline tuberculoid group the risk of serious deformity is considerably greater than in the borderline lepromatous group. Only in case of reactions is the nerve damage in the latter early and serious.

Thirdly, the borderline lepromatous patient may resemble lepromatous leprosy. Such patients are unsuitable for drug trials as the response to treatment is much better and even spontaneous remissions occur.

There is some evidence, that the response to treatment of non diffuse lepromatous patients is better on the average than of pure diffuse patients.

On the other hand, the risk of deformity, particularly after reactions is higher in the nondiffuse patients.

Pure diffuse patients, may even after a long, history of the disease and repeated ENL-reactions have good hands and good feet. There seems to be an indication that the incidence of leprosy reactions also is higher in the non-diffuse patients.

The basis of the classification proposed in this article was used for many years by the author for private purposes and proved to be more satisfactory than international classification.

It was found that other workers (e.g., Ridley and Jopling) independently had thought along the same lines. 
Those who have adopted the use of a dimorphous group will have no difficulty in recognising the cases which are not typically tuberculoid, or lepromatous, nor borderline.

The concept of low resistant tuberculoid leprosy was introduced some years ago in the teaching of auxilary staff in Nigeria and it was found that the difference with typical tuberculoid patients was easily understood.

It is however likely that there will be others who are not ready to accept this subdivision. Also there are several items which need more detailed study. Although every worker, consciously or unconsciously, uses differences in degree of definition, hypopigmentation, etc., on paper it sounds vague. In order to show differences more accurately patients or at least good photographs are needed.

Many fruitless discussions can be avoided if a committee of experienced workers from various countries could study and discuss cases presented with full details. This should include history, follow up, photographic documentation, lepromin test with a single batch of standardised antigen and histopathology, at intervals.

The material should be large and from many endemic areas. It could be supplied by various institutions and studied by the committee. There is no need for the members to come together often but the material could be circulated and discussed mainly by correspondence.

After publication of the results of the study the matter could be more fruitfully discussed in meetings of the International Congresses. This would require a permanent subcommittee for a period of Io - I 5 years at least, who keep touch with the institutions, participating in this study, and to ensure a proper follow up of as many cases as possible.

This time let us seriously attempt to come to an agreement.

\section{S U M M A R Y}

The spectral aspect of leprosy is emphasized. Indeterminate leprosy is regarded as a stage in the development towards intermediate forms of the disease. Indeterminate leprosy should not include secondary cases which have shown elevation of lesions, or macular lesions which show tuberculoid features, $e . g$. clearly defined edge and marked hypo-pigmentation.

The tuberculoid group is sub-divided into a truly benign, high resistant tuberculoid subgroup and a disseminated, more progressive group called low-resistant tuberculoid. The latter often produces very severe deformities. Lowresistant tuberculoid is, as usual, sub-divided into macular, minor and major varieties. Disseminated maculo-anaesthetic lesions fit into the first category. Reactional tuberculoid cases fit into the latter two varieties, according to the degree of infiltration.

The present borderline group comprises only a very small proportion of the intermediate cases. The borderline group is sub-divided into a group of patients with features at the tuberculoid side and a group with features at the lepromatous side. Borderline tuberculoid patients do not deteriorate towards lepromatous, whereas in borderline lepromatous patients the disease is progressive. In order to fit in many patients with intermediate features but without much infiltration, immunologically in the borderline range, maculoid, minor and major varieties are recognized.

Not all lepromatous patients are completely anergic. Many show evidence of a slight tissue defence. The lepromatous group is sub-divided into a non-diffuse and diffuse sub-group.

At the uttermost tuberculoid side of the spectrum a group of 'vaccination lesions' or inoculation lesions, including part of the self-healing childhood lesions (Lara), is recognised. The pure, primary diffuse lepromatous patients (Lucio) are placed at the other end of the spectrum. It is however doubted that special sub-groups are needed for these latter two categories.

This classification scheme reflects the spectrum of leprosy more clearly. The basic, international classification is maintained, the use of controversial terms and of new, unfamiliar designations is avoided. Classification does not become more difficult because the sub-division is based on the degree of tuberculoid and lepromatous, as before. Errors in classification are less essential in a six group classification as compared with a three group classification when they are limited to a shift towards the next group.

The still existing confusion about classification could be materially lessened if leprologists from various countries would be able to study extensive material from many parts of the world. The material should include clinical and anamnistic details, photographs, histology, lepromin reaction

\section{I4 Leprosy Review}


etc., and the patients should be followed up for a period of at least io years. The study could be performed mainly by correspondence. The results should be presented for further discussion to the International Congresses of Leprologists.

\section{REFERENGES}

I (Congress, Madrid) Report of Classification Committee. Mem. VI Congr. Internac. Leprol., Madrid, I953, pp. $75^{-80}$.
2 (Congress, Tokyo) Technical Resolutions. Classification. Trans. VIIth Internat. Congr. Leprol., Tokyo, $195^{8}$. Tofu Kyokai, Tokyo, I959, pp. 457-458.

3 (Congress, Rio de Janeiro) Technical Resolutions. Report of Round Table on Borderline and Indeterminate Leprosy. VIIIth Internat. Congr. Leprol., Rio de Janeiro, Sept. I 963 , pp. 478-48o.

4 LARA, C. B., NOlAsco, J. O. Self-healing, or abortive and residual forms of Childhood Leprosy and their probable significance. Int. 7. Leprosy, 25 (I956) p. 275

5 LEIKER, D. L. Low-resistant tuberculoid Leprosy. Int. 7. Leprosy, 32 ( ( 964), 4 pp. 359-367.

6 Ridley, D. S., JOPLing, w. H. A Classification of Leprosy for Research Purposes. Lepr. Rev., 33 (1962), I 19-1 28. 